\title{
Poor Outcome of Surgical Management of Acute Malfunctioning Mechanical Mitral Valve During Pregnancy. Should Centers with Limited Resources Find Different Options?
}

\author{
Abdelrahman Adel Abdelaziz Elsayed, ${ }^{1}$ Khaled Mohamed Abdelaal, ${ }^{1}$ Ayman Mohammad Abdelghaffar, ${ }^{1}$ \\ Essam Elbadry Hashim Mohamed, ${ }^{1}$ Tarek Mohamed Afifi Mahran, ${ }^{1}$ Mohsen Saber Mohammed Ahmed, ${ }^{1}$ \\ Alaa Mahmoud Ibrahim, ${ }^{1}$ Albaraa Ali Mansour ${ }^{2}$ \\ ${ }^{1}$ Sohag University Hospital and Faculty of Medicine, Sohag Governorate, Egypt; ${ }^{2}$ National Heart Institute, Cairo, Egypt
}

\section{ABSTRACT}

Background: Rheumatic heart disease (RHD) is the most common cardiac disease in pregnancy in developing countries with the mitral valve being the most affected. In this study, the results of surgical intervention in pregnant women presented with acute malfunctioning mechanical mitral valve were discussed.

Methods: All patients underwent emergency redo mitral valve replacement due to acute malfunctioning mechanical mitral valve during pregnancy in a single center between January 2005 and December 2017. These patients were retrospectively analyzed. Additionally, anticoagulation strategy before the event and outcomes for the mother and the fetus were outlined.

Results: Between 2005 and 2017, 16 pregnant women arrived in a single tertiary center with acute malfunctioning mechanical mitral valves. The mean gestational age at the time of presentation was $13.19 \pm 2.6$ weeks. Fifteen out of the 16 patients changed their anticoagulation regimen either with or without medical advice. After replacing the valve, cardiopulmonary bypass successfully was weaned in 12 patients, who were transferred to the ICU sedated and ventilated with variable doses of chemical Inotropes. The remaining 4 patients died on the table after failure of weaning from bypass. In one case, the patient developed immediate postoperative stroke with the Glasgow Coma Scale (GCS) of 7, CT brain revealed massive infarction, her fetus was not viable, she remained sedated and ventilated, and she passed away on post-operative Day 12, due to pneumonia and sepsis. Another patient, with a viable fetus, passed away on post-operative Day 1, due to low cardiac output.

Conclusion: Acute malfunctioning MHV during pregnancy represents a real dilemma to patients and caregivers. It carries

Received April 7, 2019; received in revised form August 19, 2019; accepted August 27, 2019.

Correspondence: Abdelrabman Adel Abdelaziz Elsayed, Consultant and Lecturer of Cardiothoracic Surgery, Department of Cardiothoracic Surgery, Sohag University Hospital, Sohag University, Sohag, Egypt; +966550569404 (e-mail: abdelrahmanadel@med.sohag.edu.eg). high fetal and maternal morbidity and mortality, especially in centers with limited resources. We believe that an alternative plane must be formulated for such patients to avoid devastating complications, including maternal and fetal deaths.

\section{INTRODUCTION}

Rheumatic heart disease (RHD) is the most common cardiac disease in pregnancy in developing countries with the mitral valve being the most affected [Bhatla 2003] while in western countries congenital heart diseases (CHD) account for the most common cardiac disease affecting pregnant women [Davies 2007].

In Egypt, despite scarce data, rheumatic heart diseases still are frequently seen in the young population [Elamrousy 2014]. Abdel-Moula et al estimated the prevalence of rheumatic heart diseases among school children in the Egyptian city of Alexandria to be $6.2 / 1000$ students, strongly associated with low socioeconomic status [Abdel-Moula 1998]. Considering the young age of the involved population, severe rheumatic valve lesions usually are fixed by mechanical valve replacement [Keogh 1999].

Management of pregnant women with mechanical heart valves (MHVs) is challenging. A report by the European

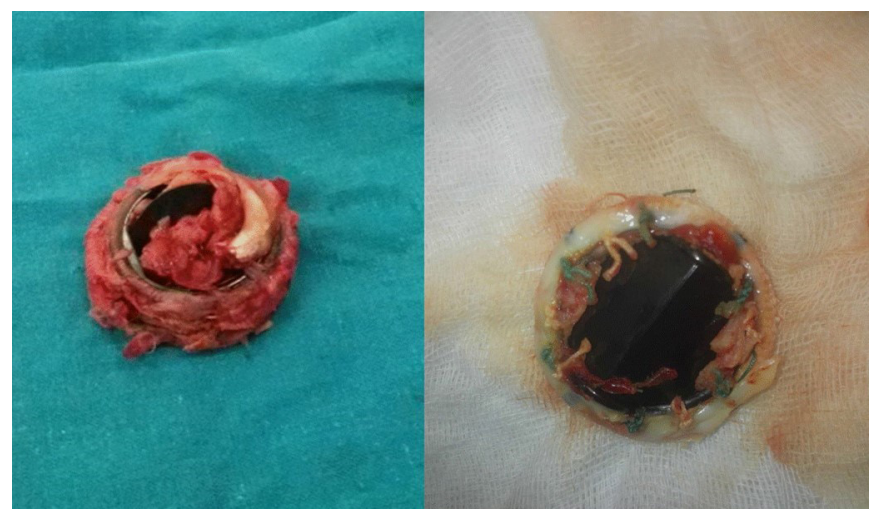

Figure 1. Mechanical valves extracted from pregnant ladies presented with acute malfunctioning mechanical mitral valves. 
Table 1. Age of Patients, Gestational Ages, Anticoagulation Strategy, Fate of the Mother and the Fetus

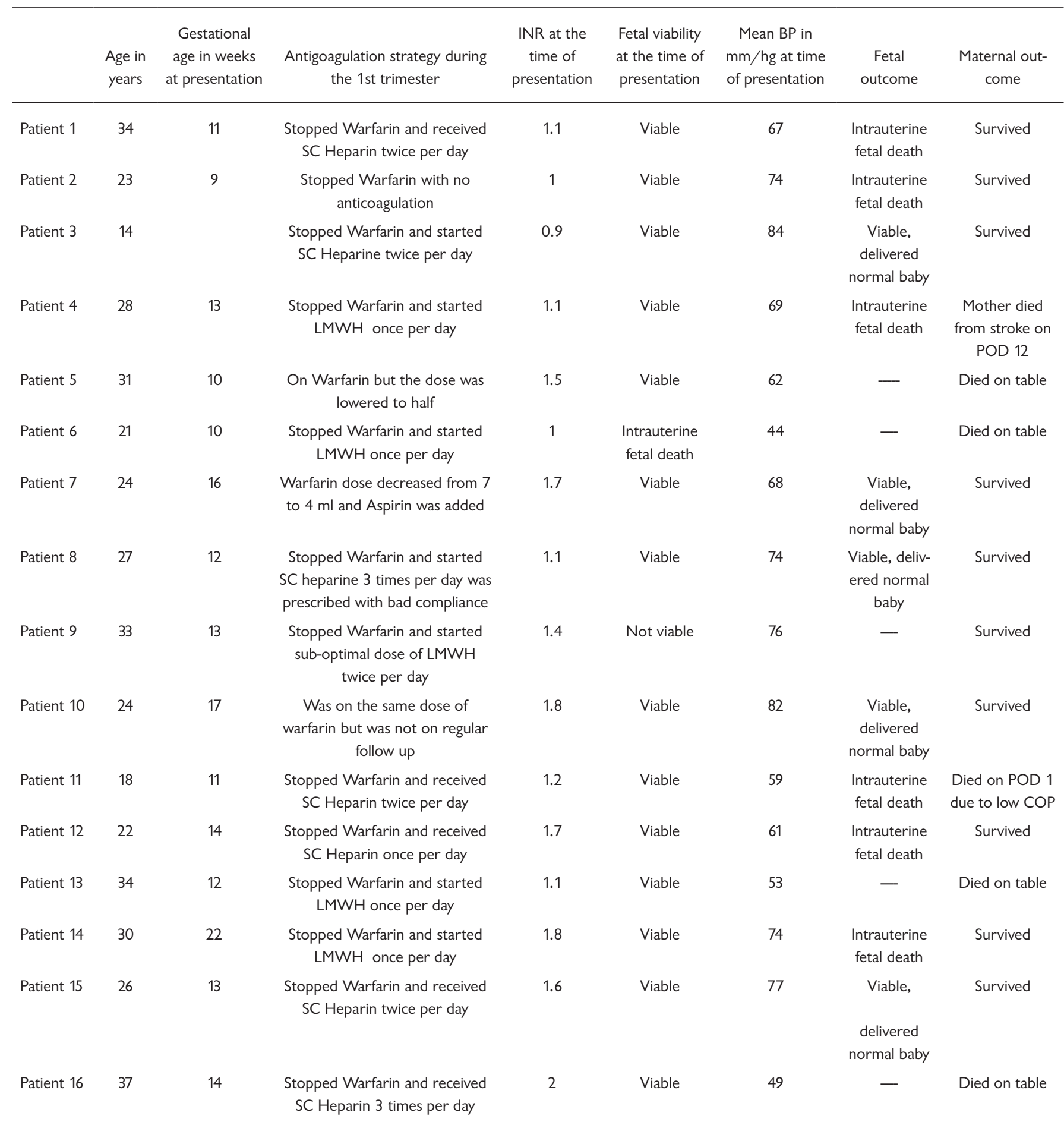




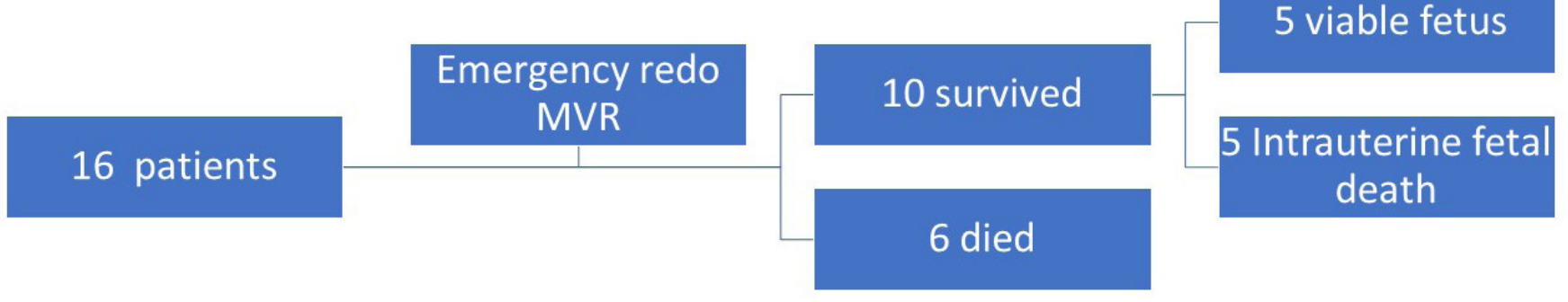

Figure 2. Outcome for the mother and fetus after emergency redo mitral valve replacement.

Society of Cardiology found that maternal mortality reached more than $4 \%$, while serious events occurred in more than $40 \%$ of pregnant women with (MHVs) [van Hagen 2015]. In another report, only $28 \%$ of pregnant women with an MHV had good maternal and fetal outcomes [Vause 2017]. Despite these facts, there is increasing social pressure on women with MHV to embark on a pregnancy in Egypt.

Pregnant women with MHVs are offered different anticoagulation options. Unfortunately, none of them is completely safe for the mother and/or fetus [van Hagen 2015; Vause 2017]. Risk-benefit evaluation must be considered to select the optimum strategy for each patient. Because of inadequate data about the safest anticoagulation regimen during pregnancy, MHV thrombosis is not infrequently encountered during pregnancy and its management carries a significant risk regardless of the used regimen [van Hagen 2015]. Furthermore, there is no standard national practice in Egypt regarding anticoagulation during pregnancy or management of malfunctioning MHVs in pregnant women.

\section{MATERIALS AND METHODS}

All patients underwent emergency redo mitral valve replacement, due to acute malfunctioning mechanical mitral valve during pregnancy in a single center between January 2005 and December 2017. These patients were retrospectively analyzed. Additionally, anticoagulation strategy before the event and outcomes for the mother and fetus were outlined.

Patients arrived at the emergency department with variable degrees of shortness of breath. Bedside transthoracic echocardiography (TTE) was obtained, and they were admitted and stabilized in the coronary care unit (CCU). Finally, the decision was made to replace the malfunctioning mechanical mitral valve. Informed high-risk consent was obtained from the patient and/or their relatives, and they were informed about the high possibility of fetal loss.

Under general anesthesia and invasive monitoring, Ketamine was used for induction, IV Tenoxicam routinely was administered as tocolytic, and anesthesia was maintained using inhalation Isoflurane. The approach in all cases was through mid-line sternotomy, occasionally, preceded by femoral triangle exposure, according to surgeon preference. Heparinization, aorto-bicaval cannulation was performed. The aorta was cross clamped and antegrade cold crystalloid cardioplegia administered. Left atriotomy or superior atrial transseptal approach was used to access the left atrium and mitral valve. Left atrial thrombus was removed, if encountered, and the mechanical valve was excised (Figure 1) and replaced with another mechanical valve in the usual manner. Cardiopulmonary bypass weaning was tried. If weaning was successful, the chest was closed, and the patient was transferred to the cardiothoracic ICU. On postoperative Day 1, fetal viability was assessed, and patients were managed, according to the usual protocol.

\section{RESULTS}

Between 2005 and 2017, 16 pregnant women arrived in a single tertiary center with acute malfunctioning mechanical mitral valves. The mean gestational age at the time of presentation was $13.19 \pm 2.6$ weeks. All patients were NYHA Class IV at the time of presentation. Mean blood pressure was above $70 \mathrm{~mm} / \mathrm{hg}$ in 7 patients and below that in 9 patients with a mean of $67.06 \pm 10.8 \mathrm{~mm} / \mathrm{hg}$. All patients were tachycardic, with a mean heart rate of $110 \pm 15$ beats/minute. Three patients presented with pulmonary edema and medically stabilized. Ten patients immediately were transferred to the OR, while 6 patients were operated on the next morning.

Fifteen out of the 16 patients changed their anticoagulation regimen with or without medical advice (Table 1); one patient was receiving her regular dose of Warfarin, but she was not regularly visiting the anticoagulation clinic.

Bedside Echo revealed a severely malfunctioning mechanical mitral valve in all patients with a mean gradient of $17.9 \pm 3.6$ $\mathrm{mm} \mathrm{Hg}$. Preoperative fetal viability revealed viable fetuses in 15 of 16 patients.

After instituting cardiopulmonary bypass and cross-clamping, the mitral valve was approached through left atriotomy in 12 patients and a superior transseptal approach in 4 patients. The mechanical valve was excised (Figure 1), any thrombi in the left atrium or left atrial appendage was removed, and the left atrial appendage was excluded if found patent. A suitably sized mechanical valve was fixed, and the atrium was closed. The tricuspid valve was repaired in 9 patients with Devega annuloplasty.

Cardiopulmonary bypass successfully was weaned in 12 patients. They were transferred to the ICU, sedated, and ventilated with variable doses of chemical Inotropes. In the remaining 4 patients, weaning from bypass was difficult, and 
Table 2. Mean Findings

\begin{tabular}{lc}
\hline & Mean \pm SD \\
\hline Age (years) & $25.9 \pm 5.5$ \\
Gestational age ( weeks) & $13.19 \pm 2.6$ \\
INR & $1.2 \pm 0.3$ \\
Mean blood pressure (mm Hg) & $67.06 \pm 10.8$ \\
Heart rate (beats/minute) & $110 \pm 15$ \\
Mitral valve mean pressure gradient (mm Hg) & $17.9 \pm 3.6$ \\
Cross clamp time (minutes) & $90.3 \pm 34$ \\
Total bypass (minutes) & $133.7 \pm 23.3$ \\
ICU stay (days) & $4.56 \pm 1.4$ \\
Hospital stay (days) & $10.4 \pm 1.6$
\end{tabular}

they couldn't maintain adequate blood pressure. The direct pulmonary artery line was inserted and mean pulmonary artery pressure was found to be high (mean of $76.7 \mathrm{~mm} / \mathrm{Hg}$ ). Inotropes including Dobutamin, Milrinone, and Noradrenaline failed to help patients to get off bypass. Transesophageal Echo revealed severely hypokinetic right ventricle in 3 patients and global hypokinesia in one patient. Unfortunately, all of them died on the table after many unsuccessful trials to get off bypass.

In the ICU, patients on average were extubated after 10.6 hours. Fetal viability was assessed on postoperative Day 1 by ultrasonography. Of the 12 patients who survived surgery, 11 had preoperatively viable fetuses. Postoperative fetal viability assessment revealed that 5 of 11 fetuses were viable, while the remaining 6 fetuses showed evidence of intrauterine fetal death (Tables 1 and 2). Patients with viable fetuses delivered normal babies by Cesarean section. In one case, the patient developed immediate postoperative stroke with the Glasgow Coma Scale (GCS) of 7, CT brain revealed massive infarction, her fetus was not viable, she remained sedated and ventilated, and she passed away on postoperative Day 12, due to pneumonia and sepsis. Another patient with a viable fetus passed away on postoperative Day 1 , due to low cardiac output (Figure 2).

\section{DISCUSSION}

American college of cardiology (ACC), American Heart Association (AHA), and European society of cardiology (ESC) pay great attention to continuous settling and modifying guidelines regarding anticoagulation strategies, during pregnancy after mechanical valve replacement [Nishimura 2017; A.T.F. Members 2012]. However, there are no guidelines, so far, about the best approach to manage pregnant women with acute malfunctioning mechanical valves.

Vitamin $\mathrm{K}$ antagonists in patients with MHV relatively are safe and effective anticoagulation [t.A.f.E.P.C., 2011]. Since pregnancy is thrombogenic [Elkayam 1996], warfarin carries a 3.9\% risk of thromboembolism in pregnant women with MHVs. The second option for anticoagulation during pregnancy is heparin, which carries a thromboembolic risk of about 33\% [Chan 2000]. It is well known that warfarin is associated with a higher risk of fetal malformations as it crosses the placenta [Xu 2016; van Hagen 2015], and the risk of embryopathy is dose-dependent; it is higher with doses of more than $5 \mathrm{mg} /$ day [Vitale 1999]. Therefore, the ESC recommended a stoppage of warfarin from 6 weeks to 12 weeks of pregnancy and using adjusted LMWH or unfractionated heparin instead, particularly with warfarin doses of more than $5 \mathrm{mg} /$ day to minimize the risk of congenital malformations [t.A.f.E.P.C., 2011].

MHVs require strict compliance with anticoagulation [Nishimura 2017] and the type of valve is to be chosen after discussion between the patient and caregivers on basis of trade-offs between benefits and hazards. For a long time, the use of biological valves for women seeking pregnancy has been advocated by some authors [Becker 1983]. More recently, the ESC reported that a desire for pregnancy is a class IIa indication for a biological valve [A.T.F. Members 2017], even though another surgery definitely would be needed. With this strategy, the patient will get the chance to have one or more pregnancies with minimal risk and one redo surgery with a reasonable risk [t.A.f.E.P.C., 2011].

During planning for the first surgery, patients were informed about the importance of adherence to the anticoagulation regimens provided by the anticoagulation clinic. A special note was given to the patient about the problem of teratogenicity associated with anticoagulation during pregnancy. Indeed, most of the ladies in this childbearing period preferred a mechanical valve to avoid another surgery. Considering the difficult access to cardiac services, their preference was justifiable. Some patients, once they got pregnant, continued their follow up with local obstetricians. In this series, all patients changed the anticoagulation regimen either without medical advice or guided by a local obstetrician. Patients generally were aware of the teratogenic effects of warfarin, and they tended to follow advice avoiding warfarin. In our anticoagulation clinic, we advocate warfarin throughout pregnancy protocol for most of the patients considering the cost of prescribing LMWH for 6 weeks and because of the unavailability of anti-Xa measurement facilities.

Acute malfunctioning MHVs in pregnant women have been infrequently discussed in literature. Some case reports demonstrated variable results [Korkmaz 2008; Oles 2001]. It is life-threatening and mostly occurs because of inadequate anticoagulation [Lengyel 2001]. In Egypt, there is no estimate of the number of pregnant women present with malfunctioning mechanical mitral valves. However, at Sohag University Hospital, 16 pregnant women presented with acute malfunctioning mechanical mitral valve between 2005 and 2017.

Diagnosis of acute malfunctioning MHV was usually straightforward; history of improper anticoagulation, typical clinical picture, and transthoracic echocardiography were enough to establish the diagnosis.

Treatment options for acute malfunctioning MHVs are either fibrinolytic therapy or surgery [Ozkan 2013]. In the 
general population, fibrinolytic therapy emerged as a promising alternative to surgery, particularly with critically ill patients [A.T.F. Members 2012]. Data regarding the safety and efficacy of fibrinolytic therapy during pregnancy is limited [A.T.F. Members 2012]. Some authors do not recommend fibrinolytic therapy during pregnancy specifically for left-sided valve thrombosis due to limited experience, risk of embolization, and sub-placental bleeding [Regitz-Zagrosek 2011]. If patients are critically ill or surgery is not immediately available, fibrinolysis should be considered [A.T.F. Members 2012]. In 2017, an AHA, ACC update of 2014 guidelines favored surgery over thrombolysis if a surgical team is available, NYHA class IV, more than an $8 \mathrm{~mm}$ clot, tricuspid valve disease, left atrial thrombus, or possible pannus [Nishimura 2017]. A very recent report concluded that complication rates were similar in pregnant and non-pregnant women receiving fibrinolytic therapy [Sousa 2018]. In light of this conflicting evidence, limited experience with fibrinolytic therapy during pregnancy and availability of a surgical team, fibrinolysis for treatment of acute malfunctioning MHV was not offered. Pregnant women with MHV thrombosis only were offered surgery.

During surgery, considering the bad general condition of patients, priority was given to maternal safety. Theoretically, high pressure, high flow, normothermic and as short as possible cardiopulmonary bypass (CPB) should be maintained [Parry 1996]. Normothermic protocol was applied to all patients. Yet, the temperature drifted to around $34^{\circ} \mathrm{C}$ after opening the sternum. Mean blood pressure was kept above $60 \mathrm{~mm} / \mathrm{hg}$. Some authors recommended fetal heart rate and uterine contraction monitoring as uterine contractions occur in response to CPB [Parry 1996]. In this series, neither uterine monitoring nor fetal heart rate monitoring was used, instead, IV tocolytics routinely were administered. Although some reports suggested that gestational age does not affect fetal mortality [Weiss 1998], others noticed a higher rate of fetal loss in patients presented at an earlier gestational age [Elassy 2014]. All patients included in this study presented before 16 weeks of pregnancy, thus, it is difficult to conclude about gestational age and fetal outcome.

In another series, fetal mortality after urgent valve replacement during pregnancy was $47 \%$ but with a mean gestational age of 25.7 weeks [Elassy 2014]. In this work, we report a slightly higher rate of fetal mortality $(50 \%)$ with a mean gestational age of 13.19 weeks. Cardiac surgery during pregnancy is associated with an acceptable maternal mortality rate especially if risk factors are well controlled [Arnoni 2003]. Maternal mortality rates were $8.7 \%$ in another study discussing emergency valve replacement during pregnancy [Elassy 2014], while in this series, maternal mortality rate significantly was higher about $37.5 \%$. We noticed that the lower the mean blood pressure at the time of presentation, the poorer the maternal outcome. While trying to explain the high mortality rate in the present series compared with others, it was noticed that patients in this series were vitally less stable, earlier in gestational age. In the community where this study was conducted, patients are among the poorest in Egypt, generally less educated, and have less access to medical services. This can explain the late presentation among this patient group.

Some studies reported the use of a right ventricular assisted device (RVAD) for patients unable to wean from cardiopulmonary bypass [Oles 2001]. Unfortunately, VAD and extracorporeal membrane oxygenation (ECMO) are not available at our center. Both would have been useful with 4 patients who died on the table because of right ventricular or bi-ventricular failure and for the patient who died a few hours after surgery because of low cardiac output (COP).

\section{CONCLUSION}

Acute malfunctioning MHV during pregnancy represents a real dilemma to patients and caregivers. It carries high fetal and maternal morbidity and mortality especially at centers with limited resources. We believe that avoiding mechanical valves for women considering pregnancy is recommended especially if poor compliance with anticoagulation is expected.

\section{REFERENCES}

Abdel-Moula, A., et al. 1998. Prevalence of rheumatic heart disease among school children in Alexandria, Egypt: A prospective epidemiological study. The Journal of the Egyptian Public Health Association 73(3-4): p. 233-254.

Arnoni, R.T., et al. 2003. Risk factors associated with cardiac surgery during pregnancy. 76(5): p. 1605-1608.

Becker, R.M. 1983. Intracardiac surgery in pregnant women. The Annals of Thoracic Surgery 36(4): p. 453-458.

Bhatla, N., et al. 2003. Cardiac disease in pregnancy. International Journal of Gynecology \& Obstetrics 82(2): p. 153-159.

Chan, W.S., S. Anand, and J.S.J.A.o.i.m. Ginsberg, 2000. Anticoagulation of pregnant women with mechanical heart valves: a systematic review of the literature. 160(2): p. 191-196.

Davies, G.A. and W.N. Herbert. 2007. HEART DISEASE IN PREGNANCY 2: Congenital Heart Disease in Pregnancy. Journal of Obstetrics and Gynaecology Canada 29(5): p. 409-414.

Elamrousy, D.M., H. Al-Asy, and W. Mawlana, 2014. Acute Rheumatic Fever in Egyptian Children: A 30-Year Experience in a Tertiary Hospital. Journal of Pediatric Sciences 6.

Elassy, S.M., A.A. Elmidany, and H.Y. Elbawab. 2014. Urgent cardiac surgery during pregnancy: a continuous challenge. The Annals of Thoracic Surgery 97(5): p. 1624-1629.

Elkayam, U. 1996. Anticoagulation in pregnant women with prosthetic heart valves: a double jeopardy. Journal of the American College of Cardiology.

Endorsed by the European Society of Gynecology , t.A.f.E.P.C., et al. 2011. ESC Guidelines on the management of cardiovascular diseases during pregnancy: the Task Force on the Management of Cardiovascular Diseases during Pregnancy of the European Society of Cardiology (ESC) 32(24): p. 3147-3197.

Lengyel, M. and L. Vandor. 2001. The role of thrombolysis in the management of left-sided prosthetic valve thrombosis: a study of 85 cases 
diagnosed by transesophageal echocardiography. The Journal of Heart Valve Disease 10(5): p. 636-649.

Keogh, B. and R. Kinsman. 1999. National adult cardiac surgical database report. London: Society of Cardiothoracic Surgeons of Great Britain and Ireland.

Korkmaz, A.A., et al. 2008. Stuck mechanical valve in pregnancy. Journal of cardiac surgery, 23(6): p. 790-792.

Members, A.T.F., et al. 2012. Guidelines on the management of valvular heart disease (version 2012) The Joint Task Force on the Management of Valvular Heart Disease of the European Society of Cardiology (ESC) and the European Association for Cardio-Thoracic Surgery (EACTS). European Journal of Cardio-Thoracic Surgery 42(4): p. S1-S44.

Members:, A.T.F., et al. 2017. 2017 ESC/EACTS Guidelines for the management of valvular heart disease. European Journal of Cardio-Thoracic Surgery 52(4): p. 616-664.

Nishimura, R.A., et al. 2017.2017 AHA/ACC focused update of the 2014 AHA/ACC guideline for the management of patients with valvular heart disease: a report of the American College of Cardiology/American Heart Association Task Force on Clinical Practice Guidelines. Journal of the American College of Cardiology 70(2): p. 252-289.

Oles, D., et al. 2001. Emergency redo mitral valve replacement in a 27-year-old pregnant female with a clotted prosthetic mitral valve, preoperative fetal demise and postoperative ventricular assist device: a case report. Perfusion 16(2): p. 159-164.

Ozkan, M., et al. 2013. Thrombolytic therapy for the treatment of prosthetic heart valve thrombosis in pregnancy with low-dose, slow infusion of tissue-type plasminogen activator. Circulation 128(5): p. 532-40.
Parry, A.J. and S. Westaby. 1996. Cardiopulmonary bypass during pregnancy. The Annals of Thoracic Surgery 61(6): p. 1865-1869.

Regitz-Zagrosek, V., et al. 2011. European Society of Gynecology (ESG), Association for European Paediatric Cardiology (AEPC), German Society for Gender Medicine (DGesGM). ESC Guidelines on the management of cardiovascular diseases during pregnancy: the Task Force on the Management of Cardiovascular Diseases during Pregnancy of the European Society of Cardiology (ESC) 32(24): p. 3147-97.

Sousa Gomes, M., M. Guimarães, and N. Montenegro. 2018. Thrombolysis in pregnancy: a literature review. The Journal of Maternal-Fetal \& Neonatal Medicine p. 1-11.

van Hagen, I.M., et al. 2015. Pregnancy in women with a mechanical heart valve: data of the European Society of Cardiology Registry of Pregnancy and Cardiac Disease (ROPAC). Circulation, p. CIRCULATIONAHA. 115.015242.

Vause, S., et al. 2017. Pregnancy outcomes in women with mechanical prosthetic heart valves: a prospective descriptive population based study using the United Kingdom Obstetric Surveillance System (UKOSS) data collection system. BJOG: An International Journal of Obstetrics \& Gynaecology 124(9): p. 1411-1419.

Vitale, N., et al. 1999. Dose-dependent fetal complications of warfarin in pregnant women with mechanical heart valves. 33(6): p. 1637-1641.

Weiss, B.M., et al. 1998. Outcome of cardiovascular surgery and pregnancy: a systematic review of the period 1984-1996. American journal of obstetrics and gynecology 179(6): p. 1643-1653.

$\mathrm{Xu}, \mathrm{Z}$, et al. 2016. Anticoagulation regimens during pregnancy in patients with mechanical heart valves: a systematic review and meta-analysis. 32(10): p. 1248. e1-1248. e9. 